\title{
An integrated clinical testing program for Filipinos afflicted with Schizophrenia
}

Datu, Jesus Alfonso $\$

De La Salle University, Manila, Philippines (jess.datu@yahoo.com)

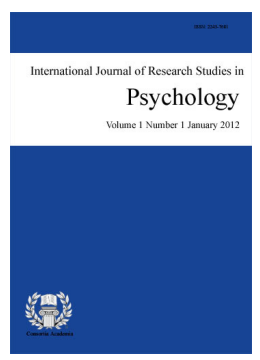

Accepted: 4 September 2012

ISSN: 2243-7681 Online ISSN: 2243-769X

OPEN ACCESS

\section{Abstract}

With the increasing number of Filipinos who are experiencing psychotic disorders, especially Schizophrenia, health professionals are being confronted with challenges related to implementation of clinically sound assessment and treatment programs. As the keystone of delineating best prognosis among the target clients, psychological assessment is a crucial step that a clinician has to be equipped with. As such, the current paper crafts a central assessment program that aims to suggest a template on how to go about evaluating psychological dimensions of the intended participants. It involves set of standardized and non-standardized assessment media to examine physiological, social and psychological substrates on the emergence of a psychotic disorder. The proposed clinical assessment program is multidisciplinary in nature which comprises psychiatrists, clinical psychologists, psychiatric nurses and psychology interns. Directions and implications of the program were charted to enhance its pragmatic utility.

Keywords: clinical assessment program; Filipino; multidisciplinary team; psychological tests; Schizophrenia 


\section{An integrated clinical testing program for Filipinos afflicted with Schizophrenia}

\section{Introduction}

Assessment program is ubiquitously renowned as an essential tool in the quest of the community of behavioral scientists to procure a better society. It constitutes a critical role of psychologists and counselors in Asian clinical and counseling settings (Chan, \& Lee, 1995; Cheung, Leong, \& Ben-Porath, 2003; Lilienfield, Wood, \& Garb, 2003). It encompasses variety of standardized psychological tests intended to probe through the psychological dimensions of different individuals. It caters to psychologists with much needed information in order to formulate a comprehensive, systematic and pragmatic analysis on the inherent capacity of individuals to spontaneously adjust to the ever-present societal transformations. Thus, development of a sound testing program as a viable form of evolutionary adaptation is vital in maintaining societal homeostasis.

By definition, a sound testing program is a dynamic course of psychometric protocol which includes series of standardized psychological test and assessment designed to depict a clearer imagery of the individual's psychological dimensions. It might also include medical and laboratory procedures especially on the case of clients who tend to manifest psychopathological symptoms such as dementia, delirium, disorganized speech, etc. As a highly integrative evaluative process, variety of professionals is needed in implementing such assessment curricula. The evaluating team may consist of clinical psychologists, psychiatrists, neurologists, psyhometricians, psychiatric nurses, occupational therapists and psychology students. Thus, the success of a particular testing program will rest heavily on the collaborative interplay between the team members.

Psychiatric disorders such as Schizophrenia have been a fascinating course of study for psychologists and allied health professionals as it primarily dwells with medically significant conditions, evidenced by the presence of psychopathology. In fact, clinical criteria specified by the Diagnostic and Statistic Manual of Mental Disorders (DSM-IV-TR) listed five characteristic symptoms of schizophrenia particularly delusions, hallucinations, disorganized speech, disorganized or catatonic behavior and "negative symptoms", meaning a reduction or loss of normal functions such as language and goal-directed behavior. This is very much congruent with Department of Health's clinical definition which states that Schizophrenia is a mental disorder characterized by profound disruption in thinking and feeling that affects behavior, as shown in the abnormality of language, thought, perception and sense of self. This includes psychotic experience like hallucinations, illusions, delusions, and disordered thinking. Additional symptoms are social withdrawal, extreme apathy, lack of drive or initiative and emotional unresponsiveness. Moreover, five sub-classification of schizophrenia were identified by $D S M-I V-T R$ namely paranoid type, disorganized type, catatonic type, undifferentiated type and residual type.

Cases of mental illness in the country are on the rise with the continuous increase in population. In line with this statement, Dr. Noel Reyes, a psychiatrist at the National Center for Mental Health in Mandaluyong City, said one of the most common brain diseases among Filipinos is schizophrenia, which afflicts one percent of the total population. In one of his recent health forums which took place last January 2007, Dr. Reyes claimed "It (cases of mental illness) increases as the population increases. For example for schizophrenia, if we are now 88 million, expect that 880,000 of us are schizophrenic." This proved to be a very alarming number as it significantly reduces the productivity rate of every individual in the society, subsequently, intensifying the dreaded socioeconomic condition of the country. More so, men and women can be equally affected with this psychiatric condition. However, onset for men is earlier, usually at 15-25 years of age. In women, age of onset is usually at 25-35 years of age. It is rare to have schizophrenia before 15 years old and after 50 years old. It has been observed, though, that men are more likely to have negative symptoms.

The most common testing program in diagnosing and evaluating the psychopathological course of Schizophrenia includes psychiatric history and mental status examinations. Psychiatric history pertains to the 
aftermath of a thorough analysis on the content of the interview process which is usually done by a medical doctor. The interview mainly comprises the patient's identification such as age, sex, marital status, occupation, and housing arrangements. Next to the exploration of aforementioned personal information is the presentation of current illness. This may consist of the past psychiatric and medical history in relation to the particular case at hand. Thereafter, the developmental, occupational and relationship history of the patient will be taken into consideration. On the other hand, mental status examination is a standard protocol primarily designed to obtain evidence of symptoms and signs of mental disorders, including dangerousness to self and others, that are present at the time of the interview. Further, evidence is obtained regarding the patient's insight, judgment, and capacity for abstract reasoning to inform decisions about treatment strategy and the choice of an appropriate treatment setting. Thus, the mental status examination is a systematic collection of data based on observation of the patient's behavior while the patient is in the psychiatrist's view during, before, and after the interview. During the mental status examination, the patient might also mention past symptoms and signs, but these should be recorded under the history of the present illness.

Bearing such considerations in mind, the proponent now has the heading. In the efforts to further bring light on the case of diagnosing and evaluating the prevalence of Schizophrenia in the country and to alleviate the negative social label that it continuously proliferates, a testing program is therefore a concern that must be given due attention. The proponent formulated a testing program that will systematically delve on the psychological dimensions of individuals afflicted with this mental illness which can be traced on the normative need to develop a specialized and multimodal testing program. This testing curriculum is a major response to the growing number of Filipino schizophrenics. Specifically, advocate of this testing curriculum employed an integral and innovative approach in assessing the psychiatric condition of patients diagnosed with this type of mental disorder in order to let these psychologically challenged individuals realize that "life is worth living" despite their psychological state. In this way, proper formulation and delivery of treatment programs can be perpetuated by looking at how biological, psychological and social substrates can contribute to the development of this psychiatric condition.

\section{Target population}

About twenty four million people have been diagnosed of Schizophrenia across different countries worldwide (World Health, Organization, 2012). With this relatively high prevalence rate, 50\% of these people were not receiving suitable mental health care services. What makes is quite alarming is the fact that $90 \%$ of these patients were coming from developing countries. Despite the presence of pharmacological and psychological treatment modalities that can be offered to patients afflicted with this condition, economic status of their respective countries would play an essential role in the process of developing sound treatment programs especially in third world countries.

Schizophrenia is a psychopathological condition characterized by perceptual distortions, thought content and process problems, reduction of will and antisocial behavioral tendencies (Lakhan \& Vieira, 2009). It includes different forms of perceptual disturbances in the form of hallucination, thinking pattern deficiencies in the form of incoherence and sometimes paranoid tendencies. Other symptoms are usually present depending on its type like paranoid, disorganized, catatonic, residual and undifferentiated. As such, combinations of neurological, psychological and behavioral disturbances maybe present among individuals who are diagnosed of this psychiatric condition. A good number of studies were executed to look at the psychopathology of schizophrenia (Lakhan \& Vieira, 2009; Siever \& Davis, 2004; Pelizza \& Ferrari, 2009).

The target population for this testing program is the Schizophrenic patients in a government mental health institution located in Metro Manila. There are about one hundred fifty (150) residents at the said institution which provides health and social welfare service for female individuals afflicted with Schizophrenia of varying types such as catatonic, disorganized, paranoid, undifferentiated and residual. Majority of these individuals are victims of traumatic experiences such as physical, sexual and psychological abuses. 


\section{Objectives}

\subsection{General objectives}

$>\quad$ To devise a testing program that will delve on the psychological dimensions of schizophrenics regardless of the classifications (catatonic, disorganized, paranoid, undifferentiated and residual types) where these psychologically challenged individuals belong and to probe through their medical status as a correlate of their functional productivity.

$>\quad$ To form a basis for establishing evidence-based therapeutic interventions.

\subsection{Specific objectives}

$>\quad$ To describe the psychological state of schizophrenics through set of standardized protocols such as mental status examination, drawing tests, etc.

$>\quad$ To assess the medical condition of the target population which includes the physician's diagnostic findings as revealed by medical examinations and clinical impressions perceived by clinical psychologists.

$>$ To administer series of tests that will delineate the course of progression of the schizophrenic's psychiatric condition.

\subsection{Multidisciplinary team}

$>\quad$ Clinical Psychologists - responsible for assessing the patient's needs, abilities or behavior using a variety of methods, including psychometric tests, interviews and direct observation.

$>$ Medical Doctor/Psychiatrists - accountable for the administration of various medical procedures such as physical examination, composition of psychiatric history, diagnosis of diseases and evaluation psychiatric condition of the patient.

$>$ Psychiatric nurse - liable for assisting the medical doctor/psychiatrist in the supervision of medical protocols needed to monitor the physical and psychiatric condition of the patient.

$>$ Social Worker - responsible for the preparation of venue and paraphernalia needed in the implementation of the proposed testing program.

$>\quad$ Psychology Intern - helps the clinical psychologist in making psychological assessment report of the patient which includes psychological tests, interview and observation.

\subsection{Materials needed}

$>\quad$ Thematic Apperception Test (TAT) - a projective test which is compose of series of pictures needed to reveal the patient's underlying depression, suicidal thoughts and aggressive impulses (Anastasi, 1986).

$>\quad$ Machover Draw a Person test (DAP) - a projective test used in revealing self-expression and other relevant information about the patient. This test is usually administered by a psychologist (Anastasi, 1986).

$>$ Calgary Depression Scale for Schizophrenia - a test specifically developed to assess the level of depression in schizophrenia (Addington \& Addington, 1990).

$>$ Stethoscope - is an acoustic medical device for auscultation, or listening to the internal sounds of a 
human body. It is often used to listen to heart sounds.

$>$ Drawing Materials - consist of pencils and bond papers that can be use in the administration of projective tests such as Draw a Person Test, and many others.

> Mental Status Exam (MSE) - is an important part of the clinical assessment process in psychiatric practice. By employing MSE, medical doctor/psychiatrist can probe through the mental state of the patient (Trzepacz \& Baker, 1993).

$>\quad$ Positive and Negative Syndrome Scale (PANNS) - assess the severity of schizophrenia symptoms and to monitor the speed and degree of a patient's response to treatment.

$>$ Medical Thermometer - is utilized for measuring human body temperature, with the tip of the thermometer being inserted either into the mouth (oral temperature), under the armpit (axillary temperature), or into the rectum via the anus (rectal temperature). It is essential for assessing the medical status of the schizophrenic patients.

$>\quad$ Sphygmomanometer - is a tool used to gauge blood pressure which is composed of an inflatable cuff to restrict blood flow, and a mercury or mechanical manometer to measure the pressure. It will help the medical doctor in determining the cardiovascular condition of the patient.

$>$ Digital Timer - is a specialized type of clock which gauges time with high degree of precision.

\section{Lists of assessment tools}

\subsection{Mental Status Examination (MSE)}

Mental status examination is a protocol given to obtain evidence of symptoms and signs of mental disorders, including dangerousness to self and others, that are present at the time of the interview. Furthermore, evidence is obtained regarding the patient's capacity for abstract reasoning to inform decisions about treatment strategy and the choice of an appropriate treatment setting. Thus, the mental status examination is a systematic collection of data based on observation of the patient's behavior while the patient is in the psychiatrist's view during, before, and after the interview. During the mental status examination, the patient might also mention past symptoms and signs, but these should be recorded under the history of the present illness. Specifically, the domains involved in MSE are patient's appearance, attitude, behavior, mood and affect, speech, thought process, thought content, perception, cognition, insight and judgment (Trzepacz \& Baker, 1993).

\subsection{Thematic Apperception Test (TAT)}

Thematic Apperception Test (TAT) is a projective technique which consists of 19 cards containing vague pictures in black and white and one blank card. The examinee is asked to make up a story to fit each picture, telling what led up to the event shown in the picture, describing what is happening at the moment and what the characters are feeling and thinking, and giving the outcome. In the case of a blank card, the examinee is instructed to imagine some picture on the card, describe it, and then tell a story about it. TAT includes an inquiry phase to clarify ambiguous responses. Then through a complex scoring system, the individual's responses are converted into an interpretation of his or her unconscious conflicts or motivation (Alloy \& Riskino, 2004).

\subsection{Machover Draw a Person Test (DAP)}

Machover Draw a Person Test is a projective technique where the examinee is provided with paper and pencil, and told simply to "draw a person". Upon the completion of the first drawing, he is asked to draw a person of the opposite sex from that of the first figure. While the individual draws, the examiner notes his 
comments, the sequence in which different parts are drawn, and other procedural details. The drawing may be followed by an inquiry in which the examinee is asked to make up a story about each person drawn, "as if he were a character in a play or novel". A series of questions is also employed during the inquiry to elicit specific information about age, schooling, occupation, family and other facts associated with characters portrayed (Anastasi, 1986).

\subsection{Calgary Depression Scale}

Calgary Depression Scale is a psychological test used in the assessment of depressive symptoms separate from positive, negative and extra-pyramidal symptoms in people with schizophrenia. The Calgary Depression Scale for Schizophrenia has been developed by Drs. Donald and Jean Addington at the University of Calgary. The Scale was specifically developed to assess the level of depression in schizophrenia. This is a nine-item scale which is answered on a zero to three metric. It has been extensively evaluated in both relapsed and remitted patients and appears sensitive to change (Addington \& Addington, 1990).

\subsection{Electrocardiograph}

Electrocardiograph $(E C G)$ is a transthoracic interpretation of the electrical activity of the heart over time captured and externally recorded by skin electrodes. It is a noninvasive recording produced by an electrocardiographic device. The electrical waves can be measured at selectively placed electrodes (electrical contacts) on the skin. Electrodes on different sides of the heart measure the activity of different parts of the heart muscle. An ECG displays the voltage between pairs of these electrodes, and the muscle activity that they measure, from different directions, also understood as vectors. This display indicates the overall rhythm of the heart and weaknesses in different parts of the heart muscle. It is the best way to measure and diagnose abnormal rhythms of the heart, particularly abnormal rhythms caused by damage to the conductive tissue that carries electrical signals, or abnormal rhythms caused by levels of dissolved salts (electrolytes), such as potassium, that are too high or low (Braunwald, 1997).

\subsection{Positive and Negative Syndrome Scale (PANNS)}

The Positive and Negative Syndrome Scale (PANSS) was developed and standardized for typological and dimensional assessment of schizophrenic phenomena. The PANSS is also extensively used to evaluate symptomatology in other psychotic disorders, including personality disorders, affective psychoses, mania, and substance related psychopathology. This scale utilizes a standard interview procedure in the form of a semi-structured clinical interview (SCI-PANSS) and an informant questionnaire (IQ-PANSS) to assure reliability. Psychologists, psychiatrists, clinical social workers, psychiatric nurses, and other mental health professionals can use the PANSS scale to assess the severity of schizophrenia symptoms and to monitor the speed and degree of a patient's response to treatment. The PANSS scale is based on findings that schizophrenia comprises at least two distinct syndromes. The positive syndrome consists of productive symptoms such as hallucinations and delusions; the negative syndrome consists of deficit features such as blunted affect and passive social withdrawal (Kay, Fiszbein, \& Opler, 1987).

\subsection{Physical examination}

The physical examination is a process by which a health care provider specifically, a medical doctor or a nurse investigates the body of a patient for signs of disease. Although health care professionals have varying approaches as to the sequence of body parts, a systematic examination generally starts at the head and finishes at the extremities. After the main organ systems have been investigated by inspection, palpation, percussion and auscultation, specific tests may follow if a particular disease is suspected. Physical examination includes evaluation of general appearance of the patient. It is recorded in the medical record in a standard layout which facilitates others later reading the notes. In addition to that, vital signs of temperature examination, pulse and blood 
An integrated clinical testing program for Filipinos afflicted with Schizophrenia pressure are usually measured. Height and weight are also gauged to identify the Body Mass Index (BMI) of the patient subsequently; ruling out obesity which can highly suggests cardiovascular anomalies.

\section{Table 1}

Timetable for test program implementation

\begin{tabular}{|c|c|c|c|}
\hline $\begin{array}{l}\text { Week } \\
\text { No. }\end{array}$ & Objectives & $\begin{array}{l}\text { Test to be used and persons } \\
\text { responsible }\end{array}$ & Outcome Indicator \\
\hline 1 & $\begin{array}{l}\text { To determine the possible } \\
\text { physiological disturbances or } \\
\text { diseases that have taken place prior } \\
\text { to the diagnosis of schizophrenia } \\
\text { To have a general overview of the } \\
\text { medical condition of the patients }\end{array}$ & $\begin{array}{l}\text { Physical Examination } \\
\text { ECG } \\
\text { (Psychiatrist or Physician, } \\
\text { Psychiatric Nurse) }\end{array}$ & $80 \%$ of the population \\
\hline 2 & $\begin{array}{l}\text { To evaluate the psychological state } \\
\text { of the patients } \\
\text { To become cognizant of the } \\
\text { underlying messages of the } \\
\text { unconscious of the patients }\end{array}$ & $\begin{array}{l}\text { Mental Status Exam } \\
\text { TAT } \\
\text { (Clinical Psychologist and } \\
\text { Psychology Intern) }\end{array}$ & $80 \%$ of the population \\
\hline 3 & $\begin{array}{l}\text { To know how the schizophrenic } \\
\text { perceives his/her self } \\
\text { To verify the extent of depression of } \\
\text { the patients }\end{array}$ & $\begin{array}{l}\text { DAP } \\
\text { Calgary Depression Scale } \\
\text { (Clinical Psychologist and } \\
\text { Psychology Intern) }\end{array}$ & $80 \%$ of the population \\
\hline 4 & $\begin{array}{l}\text { To evaluate the patients' degree of } \\
\text { sociability } \\
\text { To assess the positive and negative } \\
\text { symptoms exhibited by the patients }\end{array}$ & $\begin{array}{l}\text { Cheek and Buss Sociability Scale } \\
\text { PANSS } \\
\text { (Clinical Psychologist and } \\
\text { Psychology Intern) }\end{array}$ & $80 \%$ of the population \\
\hline
\end{tabular}

\section{Program overview and accountability}

The physical examination is a process by which a health care provider specifically, a medical doctor or a nurse investigates the body of a patient for signs of disease. Although health care professionals have varying approaches as to the sequence of body parts, a systematic examination generally starts at the head and finishes at the extremities. After the main organ systems have been investigated by inspection, palpation, percussion and auscultation, specific tests may follow if a particular disease is suspected. Physical examination includes evaluation of general appearance of the patient. It is recorded in the medical record in a standard layout which facilitates others later reading the notes. In addition to that, vital signs of temperature examination, pulse and blood pressure are usually measured. Height and weight are also gauged to identify the Body Mass Index (BMI) of the patient subsequently; ruling out obesity which can highly suggests cardiovascular anomalies.

The testing program adheres to the ethical guidelines of the American Psychiatric Association. As part of the standard protocols done in a clinical setting, the proponent utilized various medical and psychological tests to procure a much clearer imagery of the physical and psychological state of the intended populace. Moreover, proponent's criteria of selecting psychological tests were primarily based on the needs of the target population and the ability of these tests to encompass characteristics of good tests, specifically upon dwelling into the issue of validity, reliability and practicability. The latter proved to be very significant in the process of selection as the aforementioned characteristics will substantially justify the necessity for such tests to fall within the inclusion criteria. By validity, the advocates of this testing curriculum have taken into account the degree to which a test measures what is supposed to measure (Anastasi, 1986). On the other hand, reliability was considered to assess the accuracy of the measurement entailed by a particular test. Last but not the least is practicability or the extent to which a test can be used without undue expenditure of time, effort and money. In this way, the proponents can look up into the progression of the psychiatric condition of these individuals and how it would significantly affect their level of functioning.

Administrators of the program have full responsibility for the interests of the target population. It is 
important to note that various professional disciplinary fields are at stake on this endeavor and being such, paramount consideration of this liability is rigorously monitored. In fact, medical doctors, psychiatric nurses and social workers who will be taking part in this testing curriculum are proven to be professionally competent since they have passed the Professional Regulatory Commission (PRC) Licensure Examinations. Beneficence (proliferation of beneficial consequences) and non-imposition of detrimental aftermaths have been given utmost consideration to ensure the welfare of the target population. Furthermore, the multidisciplinary team is purposively selected to meet the demands of these psychologically challenged individuals. It is the duty of the professionals involved to formulate comprehensive, systematic and objective assessments of the tests that will be administered among the patients. It is also noteworthy that evaluation of the program will be done semi-annually to assess the entire testing curriculum.

Informed consent is not needed in the administration of various medical and psychological tests since the target population possessed impaired psychological state which could possibly jeopardize the rationale of legally informing them about the nature and purpose of the tests to be conducted. The proponent also respect the ultimate decision of the patient at hand, although the professionals involved in this testing program were given certain degree of freedom to choose appropriate strategies in carrying out their specific tasks.

The advocate of this testing syllabus will strictly observed the patients' confidentiality and privacy. The administration and interpretation of the test by the professionals is unveiled only when the situation calls for its disclosure such as when the law constituted to do so through a court order or if the diagnostic findings might possibly induce massive hazard on other patients. Results of the tests could be made known to the patients' immediate relatives upon legal request to the Medical Record Section of the said institution. Upon compliance to previously mentioned procedure, the medical doctor along with the psychologist will parsimoniously elucidate their diagnostic findings as revealed by the results of medical and psychological tests.

\subsection{Future thrusts}

The advocate of this testing syllabus will strictly observed the patients' confidentiality and privacy. The administration and interpretation of the test by the professionals is unveiled only when the situation calls for its disclosure such as when the law constituted to do so through a court order or if the diagnostic findings might possibly induce massive hazard on other patients. Results of the tests could be made known to the patients' immediate relatives upon legal request to the Medical Record Section of the said institution. Upon compliance to previously mentioned procedures, the medical doctor along with the psychologist will parsimoniously elucidate their diagnostic findings as revealed by the results of medical and psychological tests. The proposed testing curriculum is collaborative in nature as well given that it allows psychologists to work along with other professionals to ensure that proper therapeutic services could be given to the target populace. That being said, it can be surmised that the goal of symptom reduction among Filipino schizophrenic patients will be attained.

The proposed testing curriculum is an attempt to ameliorate the medical and psychological status of the target population. By utilizing an integrated approach in assessing the psychological dimensions of these mentally-challenged individuals, the proponents are looking forward into the continuous proliferation of various testing programs aimed at improving the mental health of Filipino people, especially on the case of populace afflicted with Schizophrenia. This proved to be a very significant advocacy as it could actually help these individuals to be given appropriate medical and psychotherapeutic treatments, subsequently, boosting their level of functional productivity. Furthermore, this testing program can also lead to sound and exemplary researches not only in the field of Medicine but Behavioral Science as well since it can provide empirical evidences needed in the perpetuity of clinical case studies that will delve into further exploring the relatively enduring dimensions of one's metaphysical domains prior to the diagnosis of Schizophrenia. In addition to that, it can also set forth to the emergence of specific clinical intervention programs that will retain the level of functioning of schizophrenics instead of intensifying their vulnerable psychiatric condition. Consequently, this leads to the development of supportive agenda intended for the betterment of the social status of these individuals, that is, 
An integrated clinical testing program for Filipinos afflicted with Schizophrenia

looking up into "what they can do" instead of resting heavily on "what they can't do". In this way, I could entail this less fortunate populace with justice and hope despite of the existential dearth that demarcates them from the population of normally-functioning individuals.

\section{References:}

Addington, D., Addington, J., \& Schissel, B. (1990). A depression rating scale for schizophrenics. Schizophrenia Research, 3, 247-251. <http://dx.doi.org/10.1016/0920-9964(90)90005-R>

Addington, D., Addington, J., \& Maticka-Tyndale, E. (1993). Rating depression in schizophrenia: a comparison of a self report and an observer report scale. Journal of Nervous and Mental Disease, 18, 561-565. $<$ http://dx.doi.org/10.1097/00005053-199309000-00006>

Alloy, A., Riskino, J. \& Manos, M. (2004). Abnormal psychology: Current perspective (9 ${ }^{\text {th }}$ ed.). New York: Mc-Graw Hill Companies, Inc.

Anastasi, A. (1986). Psychological testing ( $3^{\text {rd }}$ ed., p. 490-509). Toronto: Macmillan Co.

Braunwald, E. (1997). Heart Disease: A Textbook of Cardiovascular Medicine (5 ${ }^{\text {th }}$ ed., p. 108). Philadelphia: W. B. Saunders Co.

Chan, D. W., \& Lee, H. B. (1995). Patterns of psychological test usage in Hong Kong in 1993. Professional Psychology: Research and Practice, 26, 292-297. <http://dx.doi.org/10.1037/0735-7028.26.3.292>

Cheek, J. M., \& Buss, A. H. (1981). Shyness and sociability. Journal of Personality and Social Psychology, 41, 330-339. <http://dx.doi.org/10.1037/0022-3514.41.2.330>

Cheung, F. M., Leong, F. T., \& Ben-Porath, Y. S. (2003). Psychological assessment in Asia: Introduction to special section. Journal on Psychological Assessment, 15(3), 243-247. $<$ http://dx.doi.org/10.1037/1040-3590.15.3.243>

Lakhan, S. E., \& Vieira, K. (2009). Schizophrenia psychopathology: Are we closer to a complete model? Annals of General Psychiatry, 8(12), 1-8.

Lilienfeld, S. O., Wood, J. M., \& Garb. H. N. (2000). The scientific status of projective techniques. Psychological Science in the Public Interest, 1, 27-66.

Lindenmayer, J. P., Kay, S. R., \& Friedman, C. (1986). Negative and positive schizophrenic syndromes after the acute phase: A prospective follow-up. Comprehensive Psychiatry, 27, 276-286. $<$ http://dx.doi.org/10.1016/0010-440X(86)90003-9>

Kay, S. R., Fiszbein, A., \& Opler, L. A. (1987). The Positive and Negative Syndrome Scale (PANSS) for schizophrenia. Schizophrenia Bulletin, 13, 261-276.

Pelizza, L., \& Ferrari, A. (2009). Anhedonia in Schizophrenia and major depression: State or trait? Annals of General Psychiatry, 8(22), 5-9.

Siever, L. J., \& Davis, K. L. (2004). The pathophysiology of Schizophrenia disorders: Perspectives from the spectrum. American Journal of Psychiatry, 161, 398-413. $<$ http://dx.doi.org/10.1176/appi.ajp.161.3.398>

Trzepacz, P. T., \& Baker, R.W. (1993). The psychiatric mental status examination. Oxford, U.K.: Oxford University Press. 
Datu, J. A. 